\title{
ESTUDO DA CINÉTICA DE PIRÓLISE DE XISTO
}

\author{
J. P. FOLTIN ${ }^{1}$, A. C. L. LISBÔA ${ }^{1}$ \\ ${ }^{1}$ Universidade Estadual de Campinas, Faculdade de Engenharia Química \\ E-mail para contato: juliana.p.foltin@gmail.com
}

RESUMO - Estudos recentes apontam que as reservas petrolíferas estão diminuindo e há um elevado consumo de petróleo em todo o mundo, buscando assim, formas alternativas que poderá se tornar, no futuro, uma importante fonte de matérias-primas, utilizadas para gerar energia. Em busca de inovações e melhorias no processamento, o xisto tornou-se um objeto de estudo em paralelo, resultando em estudos de formação de óleo e gás. Este estudo tem como finalidade o entendimento da cinética de decomposição da matéria orgânica de xisto, através da Análise Termogravimétrica e Calorimetria de Varredura Diferencial. A matéria prima foi fornecida pela Petrobras. Foram estudados diferentes tamanhos de partículas, com taxas de aquecimento para o TGA de $25^{\circ} \mathrm{C} / \mathrm{min}$ para uma temperatura final até $1000^{\circ} \mathrm{C}$ e uma temperatura final até $600^{\circ} \mathrm{C}$ para o $\mathrm{DSC}$. Os resultados mostram que a taxa de perda de massa e a energia de ativação da matéria orgânica estão relacionadas com a temperatura de pirólise e a taxa de aquecimento.

\section{INTRODUÇÃO}

Nas últimas décadas o petróleo vem sendo motivo de estudo em busca de inovações e melhoras tanto para a questão de processamento como para impactos ambientais. Nesse contexto, o xisto passa a ser um objeto de estudo em paralelo, resultando em estudos de formação de óleo e gás, assim como sua recuperação. Indústrias em todo o mundo estão investindo em estudos de produtos relacionados com petróleo, particularmente a indústria química e transporte. A produção de petróleo tornou-se limitado pela capacidade da tecnologia de extração, fazendo com que a oferta e a demanda se tornem divergente nos últimos anos, o que naturalmente leva a aumentos de preços. A incerteza dos preços do petróleo bruto atuais, seu consumo crescente no mundo inteiro e disponibilidade limitada têm motivado muitos países para explorar mais os recursos do petróleo alternativo. Com isso, fonte de energia alternativa como o xisto vem ganhando espaço de estudo, considerando o óleo de xisto um substituto natural.

De acordo com Speight (2012), depósitos de xisto são encontrados em todos os continentes e tais depósitos contêm um material sólido de hidrocarboneto (querogênio) que pode ser convertido em óleo de xisto bruto por decomposição térmica para uma avaliação da matéria orgânica.

Através de pesquisas, Hubbard e Robinson (1950) mostraram que a cinética de produção de petróleo pode ser explicada de forma mais precisa, incluindo um período de indução térmica na análise de dados. Exige-se este período de indução térmica, a fim de explicar os efeitos de aquecimento não isotérmicos nos experimentos realizados. A explicação do porque avaliar a 
cinética da decomposição do xisto é de que os parâmetros cinéticos são voltados para a matéria orgânica (querogênio e betume natural). Desta forma, as medidas dos voláteis (gás, óleo, água), betume e carbono residual são necessárias para a análise da cinética.

Dentre as avaliações de estudos cinéticos, estão as aplicações de análise térmica. De acordo com os métodos resumidos por análise térmica, os métodos de Calorimetria Diferencial de Varredura (DSC) e Termogravimetria (TGA), têm um excelente significado, Opfermann (2002). A vantagem comum de ambas as técnicas de comparação com muitos outros métodos de análise é a facilidade de preparação da amostra. Além disso, as condições de ativação podem ser facilmente variadas. $\mathrm{O}$ fato de que os parâmetros termodinâmicos (entalpia de reação) e cinéticos (parâmetros de ativação como modelo cinético) podem ser determinados ao mesmo tempo, é uma das principais vantagens do método DSC. Em contraste com a TGA, o método DSC é sempre aplicável, pois quase todas as reações produzem ou consomem calor.

Neste trabalho, a análise termogravimétrica é usada para estudar os parâmetros cinéticos de xisto, empregando curvas TGA integrais e um método cinético "model free" para calcular a energia de ativação, as taxas de conversão e, em particular, o tempo de remoção de coque em função da temperatura.

\subsection{Model-free}

Segundo Fernandes Jr (2002), a análise cinética da decomposição em estado sólido é baseado de acordo com a Equação 1:

$$
\frac{\partial \alpha}{\partial T}=k e^{-\frac{E}{R T}}
$$

Uma relação química é medida em pelo menos três taxas de aquecimento diferentes. $\mathrm{O}$ parâmetro $\beta$ e as respectivas curvas de conversão são calculados fora do TG.

Para cada conversão $\alpha, \ln \left(\frac{\beta}{T^{2}}\right)$, representa uma relação gráfica $\left(\frac{1}{T}\right)$, obtendo uma reta inclinada $-\left(\frac{E a}{R}\right)$, sendo assim Ea obtida como função de conversão. Tomando a equação de taxa de reação, $\mathrm{f}(\alpha)$ e dividindo-a pela taxa de aquecimento $\beta=\frac{d T}{d t}$, obtemos a Equação 2 :

$$
\frac{\partial \alpha}{\partial t}=k f(\alpha)
$$

Rearranjando temos: 


$$
\frac{\partial \alpha}{\partial t}=\frac{k}{\beta} f(\alpha)
$$

Em que:

$\frac{\partial \alpha}{\partial t}=$ taxa de reação $\left(\mathrm{s}^{-1}\right)$

$\mathrm{k}=$ constante de velocidade $\left(\mathrm{s}^{-1}\right)$

$\alpha=$ conversão

$\beta=\frac{d T}{d t}=$ taxa de aquecimento $\left(\mathrm{k} \cdot \mathrm{s}^{-1}\right)$

Substituindo k pela expressão de Arrhenius:

$$
k=k_{0} e^{-\frac{E}{R T}}
$$

Rearranjando, temos:

$$
\frac{1}{f(\alpha)} \partial \alpha=\frac{k_{0}}{\beta} e^{-} \frac{E}{R T} \partial T
$$

Integrando-se a conversão, $\alpha$ (à temperatura T), temos:

$$
\int_{0}^{\alpha} \frac{1}{f(\alpha)} \partial \alpha=g(\alpha)=\frac{k_{0}}{\beta} \int_{T 0}^{T} e^{-\frac{E}{R T}} \partial T
$$

Uma vez que $\frac{E}{2 T}>>1$, a integral da temperatura se aproxima de acordo coma equação 6 .

$$
\int_{T 0}^{T} e^{-\frac{E}{R T}} \partial T \sim \frac{R}{E} T^{2} e^{-\frac{E}{R T}}
$$

Substituindo a temperatura integral com Ln, temos a equação 8 utilizada para determinar a Energia de Ativação para todos os valores de conversão $(\alpha)$.

$$
\ln \frac{\beta}{T^{2}}=\ln \left[\frac{R k_{0}}{E_{a} g(\alpha)}\right]-\frac{E_{a}}{R T} \frac{1}{\alpha}
$$

Para definir a conversão da matéria sólida em (xisto) querogênio para produtos a partir de dados de perda de massa por TGA é calculado de acordo com equação 8: 


$$
\alpha=\frac{w_{0}-w}{w_{0}-w_{f}}
$$

Em que:

$\mathrm{w}_{0}$ é a massa inicial da amostra (mg)

w é a massa da amostra (mg)

$\mathrm{W}_{\mathrm{f}}$ é a massa da amostra após a pirólise completa (mg).

\section{MATERIAIS E MÉTODOS}

Análises parciais de dados foram realizadas num analisador TGA modelo TGA/DSC 1 Star Sytem e DSC modelo DSC1 - Star Sytem, a matéria prima foi fornecida pela Petrobras/ SIX situada na bacia do Paraná e pertence à formação Irati, localizada na cidade de São Mateus do Sul. O restante dos dados de análise termogravimétrica foram extraídos da tese de Lisbôa, (1997).

Para a realização desta análise, a cinética de degradação não isotérmica foi utilizada cerca de $10 \mathrm{mg}$ de cada amostra, sob atmosfera de nitrogênio $\left(\mathrm{N}_{2}\right)$. Os resultados experimentais de TGA e DSC foram registrados simultaneamente. As taxas de aquecimento de pirólise foram 5, $10,20,25$ e $50{ }^{\circ} \mathrm{C} \min ^{-1}$. A temperatura de análise para o estudo da energia de ativação variou entre $110^{\circ} \mathrm{C}$ até $540^{\circ} \mathrm{C}$ para o analisador TGA, com temperatura final de $1000^{\circ} \mathrm{C} \mathrm{e}$ temperatura final de $600{ }^{\circ} \mathrm{C}$ para o analisador DSC. Para a realização dos dados experimentais, a vazão de gás obtida para a análise no TGA e DSC foi de $50 \mathrm{~mL} / \mathrm{min}$ de nitrogênio.

\section{RESULTADOS e DISCUSSÕES}

É possível observar que através dos gráficos apresentados a taxa de perda de massa devido à conversão da matéria orgânica, está claramente relacionada com a temperatura de pirólise: quanto maior for a temperatura final, maior será a perda de massa. Isto é porque, a altas temperaturas, o processo de pirólise procede mais rapidamente, como pode observar nas Figuras 1 e 2.

À medida que a taxa de aquecimento aumenta, há uma mudança de temperaturas de maior elevação para a taxa máxima de decomposição. O deslocamento no pico, que é uma medida da reatividade, tem melhor visualização nas curvas das derivadas. Isso pode ser atribuído às variações na taxa de transferência de calor com a mudança na taxa de aquecimento e o tempo de curta exposição a uma temperatura com maiores taxas de aquecimento, segundo Rajeshwar (1983), assim como o efeito da cinética de decomposição de acordo com Thakur e Nuttall, (1987). Na Figura 3, é apresentada a curva obtida pelo analisador DSC. Nota-se que, para o ponto I há mudança de linha de base sem pico, par ao ponto II pico endotérmico.

A Tabela 1 apresenta alguns dados variando a taxa de aquecimento e conversão. Observa-se que, conforme aumenta a tava de aquecimento, diminui a energia de ativação. Para cada taxa de aquecimento nota-se que a temperatura e a energia de ativação são diretamente proporcionais, ou 
seja, conforme aumenta a temperatura para a mesma taxa de aquecimento, aumenta a energia de ativação.

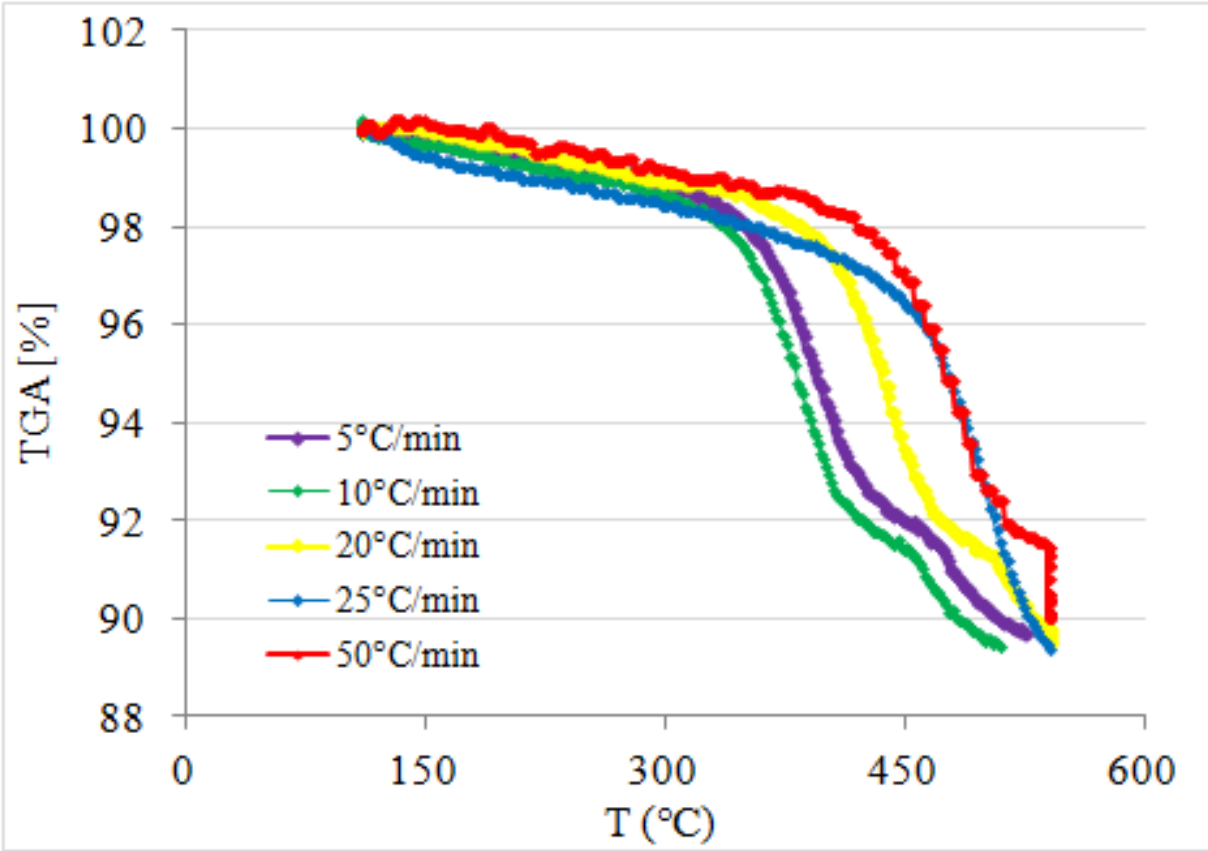

Figuras 1 - Análise obtida de xisto no analisador TGA.

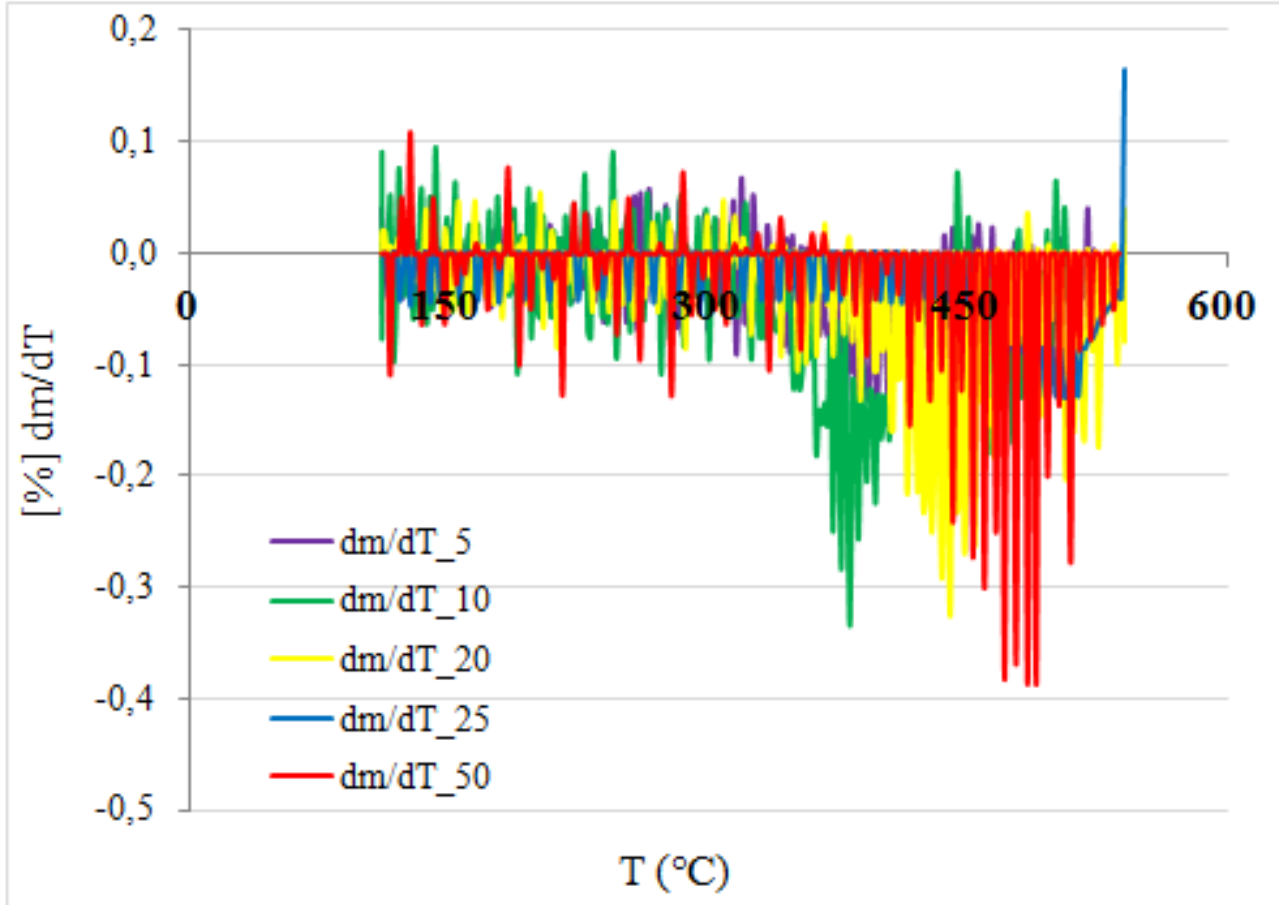

Figuras 2 - Análise obtida de xisto no analisador TGA. 


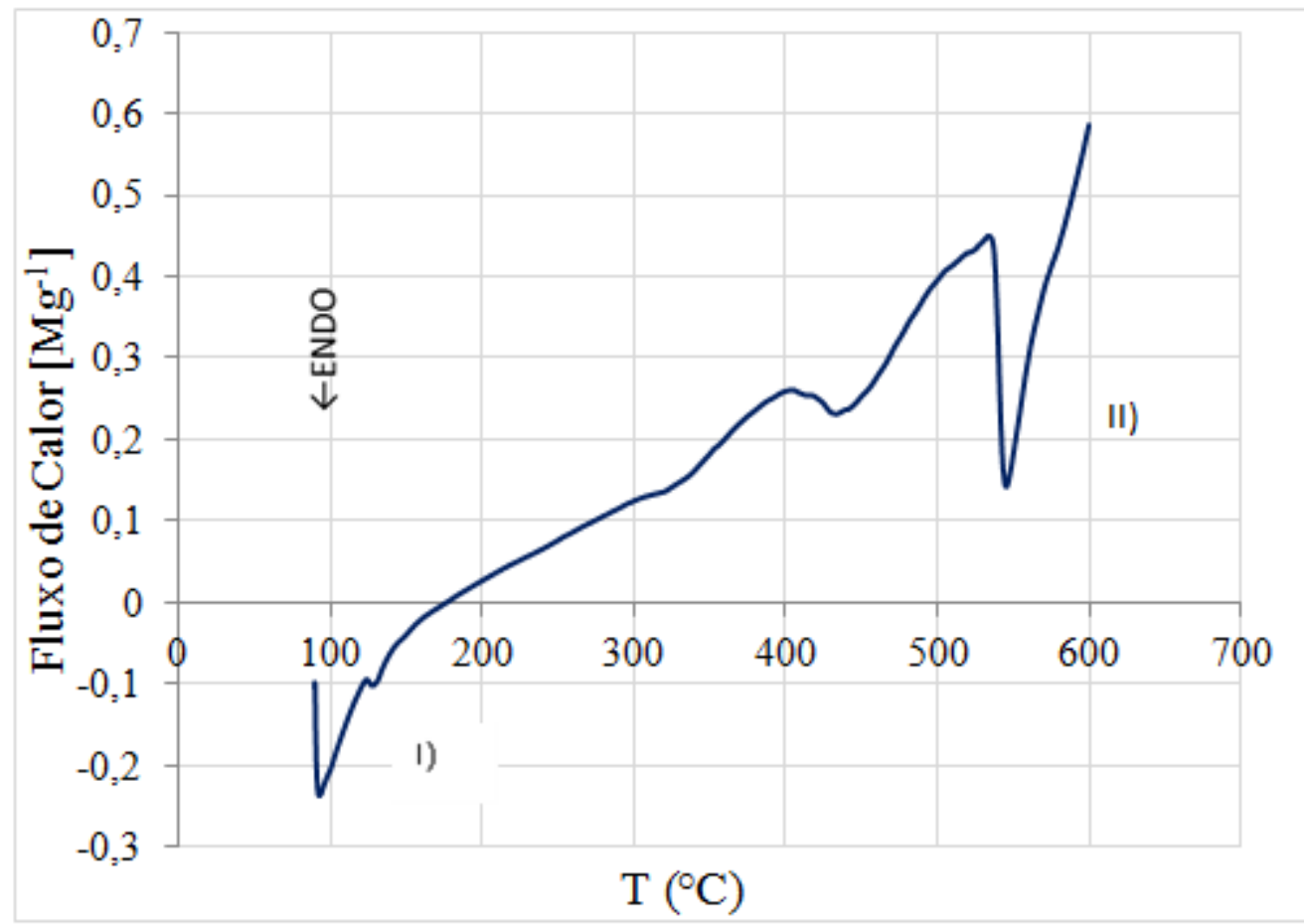

Figura 3 - Análise obtida de xisto no analisador DSC.

Tabela 1 - Dados físico-químicos obtidos das curvas TGA

\begin{tabular}{|c|c|c|c|}
\hline $\begin{array}{c}\text { Razão de } \\
\text { Aquecimento } \\
\left({ }^{\circ} \mathrm{C} / \mathrm{min}\right)\end{array}$ & $\begin{array}{c}\text { Conversão } \\
\text { [\%] }\end{array}$ & $\mathrm{T}[\mathrm{K}]$ & $\begin{array}{c}\text { Energia de } \\
\text { Ativação } \\
\text { (kJ/mol) }\end{array}$ \\
\hline \multirow[t]{2}{*}{5} & 8,9 & 328,1 & 99,8 \\
\hline & 65,4 & 524,1 & 107,6 \\
\hline \multirow[t]{2}{*}{10} & 9,1 & 243,1 & 89,2 \\
\hline & 78 & 434,2 & 98,6 \\
\hline \multirow[t]{2}{*}{20} & 9,2 & 283,4 & 86,1 \\
\hline & 77,5 & 476,5 & 94,7 \\
\hline \multirow[t]{2}{*}{25} & 9,0 & 272,5 & 82,9 \\
\hline & 75,8 & 501,3 & 93,2 \\
\hline \multirow[t]{2}{*}{50} & 9,0 & 309,2 & 79,5 \\
\hline & 76,2 & 509,2 & 87,8 \\
\hline
\end{tabular}

OBS.: o modelo cinético utiliza a unidade de temperatura em kelvin (K), portanto foi realizada a conversão dos valores das temperaturas obtidas experimentalmente (graus Celsius, ${ }^{\circ} \mathrm{C}$ ).

Avaliando a Figura 4, é possível notar que a energia de ativação é obtida a partir da Equação 7, e que, através da relação gráfica para diferentes taxas de aquecimento, resulta os dados de energia de ativação. Esta por sua vez, tem uma correlação com a taxa de conversão por uma função de potência. Para a taxa de conversão entre 20 e 100 os valores de energia de 
ativação têm um comportamento linear, com relação inversamente proporcional a taxa de aquecimento.

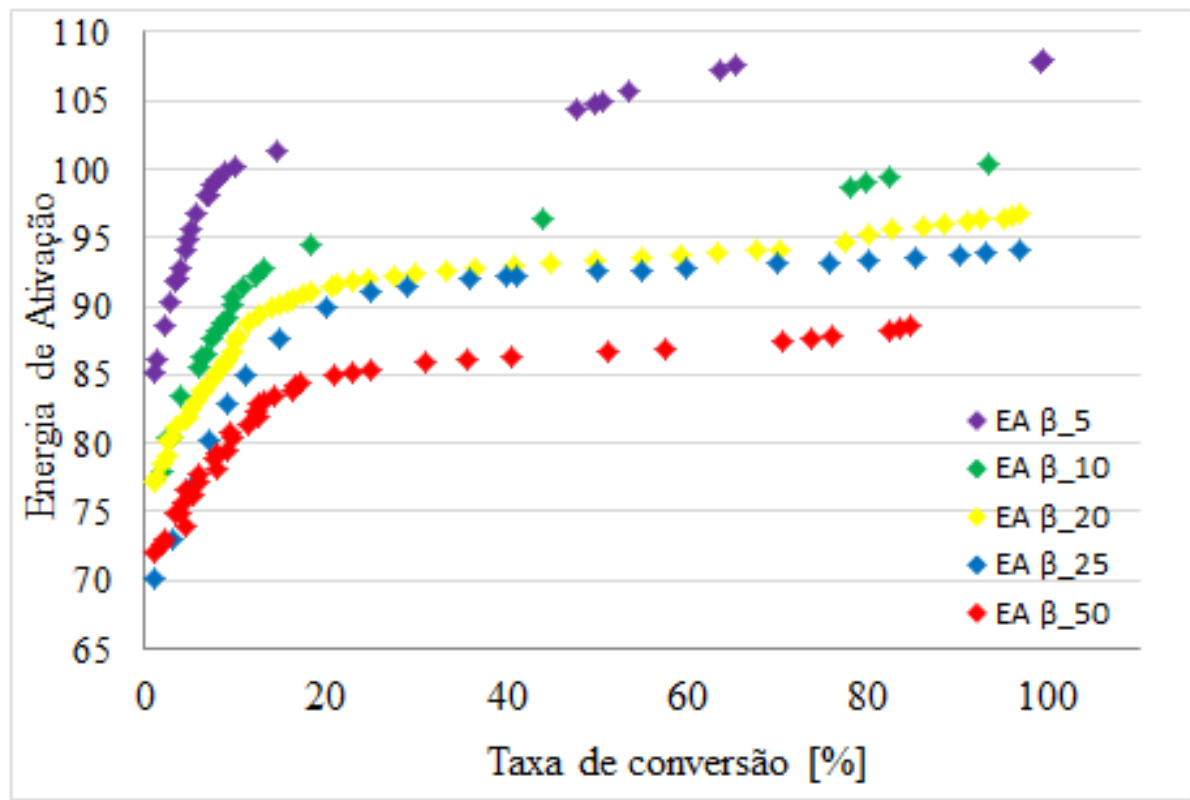

Figura 4 - Energia de ativação para diferentes taxas de aquecimento.

\section{CONCLUSÃO}

São relevantes os resultados apresentados da taxa da perda de massa em função da temperatura de pirólise. À medida que aumenta a temperatura, o processo se torna mais rápido, pois mais energia é fornecida ao sistema confirmando que a taxa de conversão diretamente proporcional a o aumento da temperatura. Em relação a energia de ativação, observa que ela aumenta em conforme a taxa de conversão cresce, tendo um comportamento de uma função de potencia e para cada taxa de aquecimento a energia de ativação é inversamente proporcional. Devido ao fato da taxa de energia fornecida ao sistema ser maior, a energia de ativação necessária é menor, configurando um comportamento é inversamente proporcional. Em futuros trabalhos pode ser avaliada a correlação da energia de ativação com a taxa de aquecimento.

\section{NOMENCLATURA}

$\mathrm{E}=$ Energia de Ativação

$f(\alpha)=$ Modelo de reação

$\mathrm{g}(\alpha)=$ Modelo de reação integrado

$\mathrm{k}(\mathrm{t})=$ Constante dependente da temperatura

$\mathrm{R}=$ Constante dos gases $\left(\mathrm{R}=8,314 \mathrm{~J} \cdot \mathrm{K}^{-1} \mathrm{~mol}^{-1}\right)$

$\mathrm{t}=$ tempo 
$\mathrm{T}=$ Temperatura

TGA = Análise Termogravimétrica

DSC $=$ Calorimetria Diferencial de Varredura

$\mathrm{w}_{0}=$ massa inicial da amostra

$\mathrm{W}=$ massa da amostra em $\mathrm{T}\left({ }^{\circ} \mathrm{C}\right)$

$\mathrm{W}_{\mathrm{f}}=$ massa da amostra após a pirólise completa

\section{Símbolos gregos}

$\alpha=$ Extensão da reação

$\beta=$ Taxa de aquecimento

\section{REFERÊNCIAS BIBLIOGRÁFICAS}

FERNANDES, Jr.; ARAÚJO, A. S.; MADRUGA, M.E.; NICOLINI L.F.; Model-free kinetics applied to regeneration of coked alumina. Elsevier, v. 392-393, p. 63-69, 2002.

HUBBARD, A B., ROBINSON, W. E., A Thermal Decomposition Study of Colorado Oil Shale, USA Bureal of Mines Report of investigations v. 4744, USA, p. $24,1950$.

LISBÔA, A. C. L. Investigation on Oil Shale Particle Reactions, Tese de Doutorado, Faculty of Chemical and Bio-Resourse Engineering, The University of British Columbia, 1997.

LISBÔA, A. C. L., WATKINSON, AP., Operating Conditions for Oil Shale Thermogravimetry, Powder Technology, v. 101, p. 151-156, 1999.

OPFERMANN, J.R.; KAISERSBERGER E.; FLAMMERSHEIM H.J.; Model-free analysis of thermoanalytical data-advantages and limitations Elsevier, v. 391, p. 119-127, 2002.

RAJESHWAR, K., Thermal analysis of coals, oil shales and oil sands, Thermochimica Acta v.63, p. $97-112,1983$.

SPEIGHT, J. G., Chapter 2 - Oil Shale Resources, Shale oil Production Processes, p. 35-73, 2012.

THAKUR, D., NUTTALL, H.E., Kinetics of pyrolysis of Moroccan oil shale by thermogravimetry, Industrial Engineering and Chemical Res. v. 26, p. 1351-1356, 1987.

\section{AGRADECIMENTOS}

Ao Conselho Nacional de Desenvolvimento Científico e Tecnológico - CNPq pelo apoio financeiro e a UNICAMP. 
19 a 22 de outubro de 2014

Florianópolis/SC 